\title{
A NOTE ON BERNOULLI NUMBERS AND SHINTANI GENERALIZED BERNOULLI POLYNOMIALS
}

\author{
MINKING EIE
}

\begin{abstract}
Generalized Bernoulli polynomials were introduced by Shintani in 1976 in order to express the special values at non-positive integers of Dedekind zeta functions for totally real numbers. The coefficients of such polynomials are finite combinations of products of Bernoulli numbers which are difficult to get hold of. On the other hand, Zagier was able to get the explicit formula for the special values in cases of real quadratic number fields.

In this paper, we shall improve Shintani's formula by proving that the special values can be determined by a finite set of polynomials. This provides a convenient way to evaluate the special values of various types of Dedekind functions. Indeed, a much broader class of zeta functions considered by the author [4] admits a similar formula for its special values. As a consequence, we are able to find infinitely many identities among Bernoulli numbers through identities among zeta functions. All these identities are difficult to prove otherwise.
\end{abstract}

\section{IDEntities AMONG Bernoulli numbers}

The Bernoulli numbers $B_{n}(n=0,1,2, \cdots)$ are defined by

$$
\frac{t}{e^{t}-1}=\sum_{n=0}^{\infty} \frac{B_{n} t^{n}}{n !}, \quad|t|<2 \pi .
$$

From the definition, one has $B_{0}=1, B_{1}=-1 / 2$. Also $B_{2 k+1}=0$ for $k \geq 1$ since the function

$$
\frac{t}{e^{t}-1}+\frac{t}{2}
$$

is an even function of $t$ by direct verification. Bernoulli numbers are used to express the special values of Riemann zeta function

$$
\zeta(s)=\sum_{n=1}^{\infty} n^{-s}
$$

namely,

$$
\zeta(2 m)=\frac{(2 \pi)^{2 m}(-1)^{m-1} B_{2 m}}{2(2 m) !}, \quad m \geq 1
$$

Received by the editors January 24, 1994 and, in reviwed form, March 2, 1995.

1991 Mathematics Subject Classification. Primary 11M41.

This work was supported by the Department of Mathematics, National Chung Cheng University, and by the National Science Foundation of Taiwan, Republic of China. 
Given the functional equation of $\zeta(s)$, this is equivalent to

$$
\zeta(1-2 m)=-\frac{B_{2 m}}{2 m} .
$$

In [10], Zagier considered the zeta function associated with a quadratic form $Q=a p^{2}+b p q+c q^{2}$ with $a, b, c>0, D=b^{2}-4 a c>0$. The zeta function is defined as

$$
Z_{Q}(s)=\sum_{p=1}^{\infty} \sum_{q=1}^{\infty}\left(a p^{2}+b p q+c q^{2}\right)^{-s}+\frac{1}{2}\left(a^{-s}+c^{-s}\right) \zeta(2 s)
$$

He proved that the special value of $Z_{Q}(s)$ at integer $-n, n \geq 0$, is given by

$$
\begin{aligned}
Z_{Q}(-n)= & \left(-\frac{B_{2 n+2}}{2 n+2}\right)\left[\int_{0}^{-b / 2 a}\left(a x^{2}+b x+c\right)^{n} d x+\int_{0}^{-b / 2 c}\left(a+b y+c y^{2}\right)^{n} d y\right] \\
& +\sum_{r=0}^{2 n} \frac{B_{r+1} B_{2 n-r+1}}{(r+1)(2 n-r+1)} d_{r, n}+ \begin{cases}-1 / 2 & \text { if } n=0, \\
0 & \text { if } n \geq 1 .\end{cases}
\end{aligned}
$$

where $d_{r, n}$ is the coefficient of $x^{r} y^{n-r}$ in $\left(a x^{2}+b x y+c y^{2}\right)^{n}$.

Remark. Zagier's formula was reproduced in van der Geer's book [5] with a slight mistake. The formula here is a corrected version.

In [4], the author considered the zeta function $Z(P, \beta ; s)$ associated with a product of linear forms

$$
P(x)=\prod_{j=1}^{n}\left(a_{1 j} x_{1}+\cdots+a_{r j} x_{r}+\delta_{j}\right), \quad \text { Re } a_{i j}>0, \operatorname{Re} \delta_{j}>0,
$$

defined as

$$
Z(P, \beta ; s)=\sum_{n_{1}=1}^{\infty} \cdots \sum_{n_{r}=1}^{\infty} n_{1}^{\beta_{1}} \cdots n_{r}^{\beta_{r}} P\left(n_{1}, \cdots, n_{r}\right)^{-s}, \quad \text { Re } s>\frac{|\beta|+r}{n} .
$$

Here $\beta=\left(\beta_{1}, \cdots, \beta_{r}\right)$ is a $r$-tuple of non-negative integers. The special value of $Z(P, \beta ; s)$ at integer $-m, m \geq 0$, can be determined by $n \cdot 2^{r}$ polynomials which are related to $x^{\beta} P^{m}(x)$. Indeed, one has

$$
Z(P, \beta ;-m)=\frac{1}{n} \sum_{1 \leq j_{1}<\cdots<j_{q} \leq r} J^{r-q}\left[\int_{\Delta_{j}\left(x_{j_{1}}, \cdots, x_{j_{q}}\right)} x^{\beta} P^{m}(x) d x_{j_{1}} \cdots d x_{j_{q}}\right] .
$$

When $a_{i j}$ and $\delta_{j}$ are real numbers, the domain $\Delta_{j}\left(x_{j_{1}}, \cdots, x_{j_{q}}\right)$ is a simplex in $\mathbb{R}^{q}$ defined by

$$
\left\{\begin{array}{l}
x_{j_{1}} \leq 0, \cdots, x_{j_{q}} \leq 0, \\
a_{1 j} x_{1}+\cdots+a_{r j} x_{r}+\delta_{j} \geq 0 .
\end{array}\right.
$$

Also for any polynomial $f(x)=\sum a_{\alpha} x_{1}^{\alpha_{1}} \cdots x_{p}^{\alpha_{p}}$ of $p$ variables,

$$
\begin{aligned}
J^{p}[f(x)] & =\sum a_{\alpha} \zeta\left(-\alpha_{1}\right) \cdots \zeta\left(-\alpha_{p}\right) \\
& =\sum a_{\alpha}(-1)^{|\alpha|} \frac{B_{\alpha_{1}+1} \cdots B_{\alpha_{p}+1}}{\left(\alpha_{1}+1\right) \cdots\left(\alpha_{p}+1\right)} .
\end{aligned}
$$

With the formula we shall derive below for special values of $Z(P, \beta ; s)$, we are able to transform identities among zeta functions into identities among Bernoulli numbers. The procedure is demonstrated in the following proposition: 
Proposition 1. For $n \geq 4$, one has

$$
\sum_{p=2}^{n-2} \frac{(2 n-2) !}{(2 p-2) !(2 n-2 p-2) !} \cdot \frac{B_{2 p}}{2 p} \cdot \frac{B_{2 n-2 p}}{2 n-2 p}=\left(-\frac{B_{2 n}}{2 n}\right) \frac{(2 n+1)(2 n-6)}{6(2 n-2)(2 n-3)} .
$$

Proof. Consider the zeta function

$$
Z(s)=\sum_{n_{1}=1}^{\infty} \sum_{n_{2}=1}^{\infty} n_{1} n_{2}\left(n_{1}+n_{2}\right)^{-s}, \quad \text { Re } s>4
$$

Let $r=n_{1}+n_{2}$ be a new variable in place of $n_{2}$. Then for Re $s>4$,

$$
\begin{aligned}
Z(s) & =\sum_{r=2}^{\infty} r^{-s} \cdot \sum_{n_{1}=1}^{r-1}\left(r-n_{1}\right) n_{1} \\
& =\frac{1}{6} \sum_{r=2}^{\infty} r^{-s}\left(r^{3}-r\right) \\
& =\frac{1}{6}[\zeta(s-3)-\zeta(s-1)] .
\end{aligned}
$$

One can now make an analytic continuation. In particular, one has for $n \geq 4$

$$
\begin{aligned}
Z(4-2 n) & =\frac{1}{6}[\zeta(1-2 n)-\zeta(3-2 n)] \\
& =\frac{1}{6}\left[-\frac{B_{2 n}}{2 n}+\frac{B_{2 n-2}}{2 n-2}\right] .
\end{aligned}
$$

On the other hand, we also have

$$
\begin{aligned}
Z(4-2 n)= & J^{2}\left[x y(x+y)^{2 n-4}\right]+J\left[\int_{0}^{-x} x y(x+y)^{2 n-4} d y+\int_{0}^{-y} x y(x+y)^{2 n-4} d x\right] \\
= & \sum_{p=0}^{2 n-4} \frac{(2 n-4) !}{p !(2 n-p-4) !} \frac{B_{p+2}}{p+2} \frac{B_{2 n-p-2}}{2 n-p-2}+\left(-\frac{B_{2 n}}{2 n}\right) \frac{2}{(2 n-2)(2 n-3)} \\
= & \sum_{p=2}^{n-2} \frac{(2 n-4) !}{(2 p-2) !(2 n-2 p-2) !} \frac{B_{2 p}}{2 p} \frac{B_{2 n-2 p}}{2 n-2 p}+\frac{1}{6} \frac{B_{2 n-2}}{2 n-2} \\
& \quad+\left(-\frac{B_{2 n}}{2 n}\right) \frac{2}{(2 n-2)(2 n-3)}
\end{aligned}
$$

Comparing the two expressions for $Z(4-2 n)$, we get our assertion.

Remark. The identity of Proposition 1 appears in [7] as a consequence of an identity among Eisenstein series of different weights:

$$
\frac{1}{6}(2 n+1)(2 n-1)(2 n-6) G_{2 n}(z)=\sum_{p=2}^{n-2}(2 p-1)(2 n-2 p-1) G_{2 p}(z) G_{2 n-2 p}(z),
$$


where $G_{2 k}(z)$ is the Eisenstein series defined by

$$
G_{2 k}(z)=\sum_{(m, n) \in \mathbb{Z}^{2}-(0,0)}(m+n z)^{-2 k}
$$

The above procedure can be applied to other zeta functions to get a lot of identities of a similar kind. Here we mention some of them.

(1) Consider the identity of zeta functions:

$$
Z_{1}(s)=\sum_{n_{1}=1}^{\infty} \sum_{n_{2}=1}^{\infty}\left(n_{1}+n_{2}\right)^{-s}=\zeta(s-1)-\zeta(s)
$$

With $s=2-2 n, n \geq 2$, one gets the classical identity due to Euler [1],

$$
\sum_{k=1}^{n-1} \frac{(2 n) !}{(2 k) !(2 n-2 k) !} B_{2 k} B_{2 n-2 k}=-(2 n+1) B_{2 n}
$$

(2) Consider the identity of zeta functions:

$$
Z_{2}(s)=\sum_{n_{1}=1}^{\infty} \sum_{n_{2}=1}^{\infty} \sum_{n_{3}=1}^{\infty}\left(n_{1}+n_{2}+n_{3}\right)^{-s}=\frac{1}{2}[\zeta(s-2)-3 \zeta(s-1)+2 \zeta(s)] .
$$

By letting $s=3-2 n$ with $n \geq 3$ and using the identity in (1), we get

$$
\sum_{\substack{p+q+r=n \\ p, q, r \geq 1}} \frac{(2 n) !}{(2 p) !(2 q) !(2 r) !} B_{2 p} B_{2 q} B_{2 r}=\frac{(2 n+1)(2 n+2)}{2} B_{2 n}+\frac{n(2 n-1)}{2} B_{2 n-2} .
$$

(3) Consider the identity of zeta functions:

$$
\begin{aligned}
Z_{3}(s) & =\sum_{n_{1}=1}^{\infty} \sum_{n_{2}=1}^{\infty} \sum_{n_{3}=1}^{\infty} \sum_{n_{4}=1}^{\infty}\left(n_{1}+n_{2}+n_{3}+n_{4}\right)^{-s} \\
& =\frac{1}{6}[\zeta(s-3)-6 \zeta(s-2)+11 \zeta(s-1)-6 \zeta(s)] .
\end{aligned}
$$

By letting $s=4-2 n$ with $n \geq 4$ and using the identities in (1) and (2), we get

$$
\begin{aligned}
\sum_{\substack{p+q+r+s=n \\
p, q, r, s \geq 1}} \frac{(2 n) !}{(2 p) !(2 q) !(2 r) !(2 s) !} B_{2 p} B_{2 q} B_{2 r} B_{2 s} \\
\quad=-\left[\frac{(2 n+1)(2 n+2)(2 n+3)}{6} B_{2 n}+\frac{4 n^{2}(2 n-1)}{3} B_{2 n-2}\right] .
\end{aligned}
$$

(4) Consider the identity of zeta functions:

$$
Z_{4}(s)=\sum_{n_{1}=1}^{\infty} \sum_{n_{2}=1}^{\infty} n_{1}^{2} n_{2}^{2}\left(n_{1}+n_{2}\right)^{-s}=\frac{1}{30}[\zeta(s-5)-\zeta(s-1)] .
$$


By setting $s=6-2 n, n \geq 4$, we get

$$
\begin{aligned}
\sum_{p=3}^{n-3} \frac{(2 n-6) !}{(2 p-3) !(2 n-2 p-3) !} & \frac{B_{2 p}}{2 p} \frac{B_{2 n-2 p}}{2 n-2 p} \\
& =\frac{(2 n+1)\left(4 n^{2}-26 n+60\right)}{30(2 n-3)(2 n-4)(2 n-5)}\left(-\frac{B_{2 n}}{2 n}\right)+\frac{1}{60} B_{2 n-4}
\end{aligned}
$$

(5) Consider the identity of zeta functions:

$$
\begin{aligned}
Z_{5}(s) & =\sum_{n_{1}=1}^{\infty} \sum_{n_{2}=1}^{\infty} \sum_{n_{3}=1}^{\infty} n_{1} n_{2} n_{3}\left(n_{1}+n_{2}+n_{3}\right)^{-s} \\
& =\frac{1}{120} \zeta(s-5)-\frac{1}{24} \zeta(s-3)+\frac{1}{30} \zeta(s-1)
\end{aligned}
$$

By setting $s=6-2 n$ with $n \geq 6$, we get

$$
\begin{aligned}
\sum_{\substack{p+q+r=n \\
p, q, r \geq 2}} \frac{(2 n-6) !}{(2 p-2) !(2 q-2) !(2 r-2) !} \frac{B_{2 p} B_{2 q} B_{2 r}}{8 p q r} \\
\quad=\left(-\frac{B_{2 n}}{2 n}\right)\left[\frac{1}{120}-\frac{2 n^{2}-5 n}{(2 n-2)(2 n-3)(2 n-4)(2 n-5)}\right]+\frac{1}{80}\left(\frac{B_{2 n-4}}{2 n-4}\right) .
\end{aligned}
$$

2. Shintani's Generalized Bernoulli POLYNOMials

The classical Bernoulli polynomials $B_{n}(x)(n=0,1,2, \cdots)$ are defined by

$$
B_{n}(x)=\sum_{k=0}^{n}\left(\begin{array}{l}
n \\
k
\end{array}\right) B_{n-k} x^{k}
$$

Here $\left(\begin{array}{l}n \\ k\end{array}\right)$ is the binomial coefficient $\frac{n !}{(n-k) ! k !}$. Equivalently, one has

$$
\frac{t e^{x t}}{e^{t}-1}=\sum_{n=0}^{\infty} \frac{B_{n}(x) t^{n}}{n !}, \quad|t|<2 \pi .
$$

Bernoulli polynomials can be used to express the special values at negative integers of Hurwitz's zeta function $\zeta(s ; x)$, defined by

$$
\zeta(s ; x)=\sum_{n=0}^{\infty}(n+x)^{-s}, \quad x>0, \operatorname{Re} s>1 .
$$

In fact, one has for $m \geq 1$,

$$
\zeta(1-m ; x)=-\frac{B_{m}(x)}{m} .
$$


For any $r \times n$ matrix $A=\left[a_{j k}\right]$ with positive entries and any $r$-tuple of complex numbers $x=\left(x_{1}, \cdots, x_{r}\right)$, Shintani considered in [6] the zeta function

$$
\zeta(s, A, x)=\sum_{n_{1}=0}^{\infty} \cdots \sum_{n_{r}=0}^{\infty} \prod_{k=1}^{n}\left[a_{1 k}\left(n_{1}+x_{1}\right)+\cdots+a_{r k}\left(n_{r}+x_{r}\right)\right]^{-s} .
$$

Set

$$
\begin{cases}L_{j}(t)=a_{j 1} t_{1}+\cdots+a_{j n} t_{n}, & 1 \leq j \leq r \\ L_{k}^{*}(u)=a_{1 k} u_{1}+\cdots+a_{r k} u_{r}, & 1 \leq k \leq n .\end{cases}
$$

Shintani's generalized Bernoulli polynomial $B_{m}(A, x)$ is defined as the special value of $\zeta(s, A, x)$ at $s=1-m$ up to a constant factor; namely,

$$
\zeta(1-m, A, x)=(-1)^{r} m^{-n} B_{m}(A, x) .
$$

In the same paper, Shintani was able to express $B_{m}(A, x)$ in terms of a combination of products of Bernoulli polynomials as follows:

$$
\begin{aligned}
\frac{B_{m}(A, x)}{(m !)^{n}}=\sum_{\substack{|p|=n(m-1)+r \\
p_{1}, \cdots, p_{r} \geq 1}} \frac{B_{p_{1}}\left(x_{1}\right) \cdots B_{p_{r}}\left(x_{r}\right)}{p_{1} ! \cdots p_{r} !} C(A, p) \\
+\frac{1}{n} \sum_{S} \sum_{\substack{|q|=n(m-1)+r \\
q_{j} \geq 1}}\left\{\prod_{j \in S} \frac{B_{q_{1}}\left(x_{j}\right)}{q_{j} !}\right\} \sum_{k=1}^{n} C(S, q, A)^{(k)} .
\end{aligned}
$$

Here $C(A, p)$ is the coefficient of $\left(t_{1} \cdots t_{n}\right)^{m-1}$ in the polynomial $\prod_{j=1}^{r} L_{j}(t)^{p_{j}-1}, S$ ranges over all non-empty subsets of $I=\{1,2, \cdots, r\}$, and $C(S, q, A)^{(k)}$ is the coefficient of $\left(t_{1} \cdots t_{k-1} t_{k+1} \cdots t_{n}\right)^{m-1}$ in the Taylor expansion of the function

$$
\prod_{j \in S} L_{j}(t)^{q_{j}-1} /\left.\prod_{j \notin S} L_{j}(t)\right|_{t_{k}=1}
$$

Such a formula is useful in calculating the special values at non-positive integers of Dedekind zeta functions of totally real number fields. However, it is typically quite painful and laborious to compute $C(A, p)$ as well as $C(S, q, A)^{(k)}$.

Here we shall prove that $\zeta(1-m, A, x)$ can be determined by $n \cdot 2^{r}$ polynomials which can be obtained from $[P(u)]^{m-1}=\prod_{k=1}^{n} L_{k}^{*}(u)^{m-1}$ by integrating over certain simplexes. We need a correspondence from polynomials to products of Bernoulli polynomials.

Given any subset $S$ of $I=\{1,2, \cdots, r\}$ and polynomial $g(u)$ with variables in $\left\{u_{i} \mid i \in S\right\}$. If

$$
g(u)=\sum_{|\alpha|=0}^{p} b_{\alpha} \cdot \prod_{i \in S} u_{i}^{\alpha_{i}}
$$

we let

$$
J_{S}[g]=\sum_{|\alpha|=0}^{p} b_{\alpha} \cdot \prod_{i \in S} \frac{-B_{\alpha_{i}+1}\left(x_{i}\right)}{\alpha_{i}+1} .
$$

When $S=\varnothing$, we let $J_{\phi}[c]=c$ for any constant $c$.

Then Shintani's formula for $\zeta(1-m, A, x)$ can be reformulated as follows: 
Theorem. In the above notation, for any positive integer $m$,

$$
\zeta(1-m, A, x)=\frac{1}{n} \sum_{S} J_{S}\left[\sum_{k=1}^{n} \int_{\Delta_{k, S}(u)} P^{m-1}(u) \prod_{j \notin S} d u_{j}\right] .
$$

Here $S$ ranges over all non-empty subsets of $I=\{1,2, \cdots, r\}$ and $\Delta_{k, S}(u)$ is the simplex defined by

$$
\Delta_{k, S}(u):\left(u_{j}\right)_{j \notin S}, u_{j} \leq 0, L_{k}^{*}(u)=a_{1 k} u_{1}+\cdots+a_{r k} u_{r} \geq 0 .
$$

To prove the theorem, we need to evaluate $C(A, p)$ and $C(S, q, A)^{(k)}$ explicitly. Here we transform them into integrals so that we can apply the results obtained in [4].

Let $E^{n}$ be the standard simplex in $\mathbb{R}^{n}$ defined by

$$
E^{n}:\left(u_{1}, \cdots, u_{n}\right) \in \mathbb{R}^{n}, u_{i} \geq 0, \sum_{i=1}^{n} u_{i}=1 .
$$

Let $d U=d u_{1} \cdots d u_{n-1}$ be the Euclidean measure on $E^{n}$.

Lemma 1. For any homogeneous polynomial $P(u)$ of degree $n(m-1)$, the integral

$$
F(s)=\frac{1}{\Gamma(s)^{n-1}} \int_{E^{n}}\left(u_{1} \cdots u_{n}\right)^{s-1} P(u) d U, \quad \operatorname{Re} s>0,
$$

as a function of $s$, has an analytic continuation to the whole s-plane. Furthermore, $F(1-m)=(-1)^{(n-1)(m-1)} n[(m-1) !]^{n-1} \times\left[\right.$ coefficient of $\left(u_{1} \cdots u_{n}\right)^{m-1}$ in $\left.P(u)\right]$.

Proof. Let

$$
P(u)=\sum_{|\beta|=n(m-1)} C_{\beta} u_{1}^{\beta_{1}} \cdots u_{n}^{\beta_{n}} .
$$

Then, for Re $s>0$, a term by term integration yields

$$
F(s)=\sum_{|\beta|=n(m-1)} C_{\beta} \frac{\Gamma\left(s+\beta_{1}\right) \cdots \Gamma\left(s+\beta_{n}\right)}{\Gamma(s)^{n-1} \Gamma(n s+|\beta|)} .
$$

For fixed $\beta=\left(\beta_{1}, \cdots, \beta_{n}\right)$ with $|\beta|=n(m-1)$, let

$$
A_{\beta}(s)=\frac{\Gamma\left(s+\beta_{1}\right) \cdots \Gamma\left(s+\beta_{n}\right)}{\Gamma(s)^{n-1} \Gamma(n s+|\beta|)} .
$$

Note that any possible poles of $\Gamma\left(s+\beta_{1}\right) \cdots \Gamma\left(s+\beta_{n}\right)$ are cancelled by the poles arising from $\Gamma(s)^{n-1} \Gamma(n s+|\beta|)$. Thus $A_{\beta}(s)$ is a regular function of $s$. Also $A_{\beta}(1-m)=0$ unless $\beta_{1}=\beta_{2}=\cdots=\beta_{n}=m-1$. For such an exceptional case, we have

$$
A_{\beta}(1-m)=(-1)^{(n-1)(m-1)} n[(m-1) !]^{n-1} .
$$

This proves our assertion.

With Lemma 1, we are able to express the coefficient $C(A, p)$ in Shintani's formula as follows. 
Corollary. For any $r$-tuple of positive integers $p=\left(p_{1}, \ldots, p_{r}\right)$ with $|p|=$ $n(m-1)+r$, one has

$$
n C(A, p)=(-1)^{(n-1)(m-1)}[(m-1) !]^{n-1} F(1-m),
$$

where for $\operatorname{Re} s>0$,

$$
F(s)=\frac{1}{\Gamma(s)^{n-1}} \int_{E^{n}}\left(u_{1} \ldots u_{n}\right)^{s-1} \prod_{j=1}^{r} L_{j}(u)^{p_{j}-1} d U \cdot \square
$$

We now proceed to consider the same type of integral as $F(s)$ but with $p_{j}=0$ for some $j$. First we need a lemma to evaluate the integral around a particular vertex of $E^{n}$. For any positive number $h<1$, we let $E_{j}^{n}(h)$ be the subset of $E^{n}$ defined by

$$
\begin{aligned}
E_{j}^{n}(h) & =\left\{\left(u_{1}, \ldots, u_{n}\right) \in E^{n} \mid 0 \leq u_{1}+\ldots+u_{j-1}+u_{j+1}+\ldots+u_{n} \leq h\right\} \\
& =\left\{\left(u_{1}, \ldots, u_{n}\right) \in E^{n} \mid 1-h \leq u_{j} \leq 1\right\} .
\end{aligned}
$$

Lemma 2. For $\operatorname{Re} s>0$ and nonegative integers $\alpha_{1}, \ldots, \alpha_{n}$, one has

$$
\begin{aligned}
\int_{E_{j}^{n}(h)} \prod_{i=1}^{n}\left(u_{i}\right)^{s+\alpha_{i}-1} d U & \\
& =\prod_{i \neq j} \Gamma\left(s+\alpha_{i}\right) \cdot \frac{1}{\Gamma\left((n-1) s+|\alpha|_{j}\right)} \int_{0}^{h} t^{(n-1) s+|\alpha|_{j}-1}(1-t)^{s+\alpha_{j}-1} d t
\end{aligned}
$$

where $|\alpha|_{j}=|\alpha|-\alpha_{j}$.

Proof. Set

$$
\left\{\begin{array}{l}
t=u_{1}+\ldots+u_{j-1}+u_{j+1}+\ldots+u_{n} \\
u_{i}^{\prime}=\frac{u_{i}}{t} \quad(i \neq j)
\end{array}\right.
$$

Then $u^{\prime}=\left(u_{1}^{\prime}, \ldots, u_{j-1}^{\prime}, u_{j+1}^{\prime}, \ldots, u_{n}^{\prime}\right) \in E^{n-1}$ and

$$
\frac{\partial\left(t, u_{1}^{\prime}, \ldots, u_{n}^{\prime}\right)}{\partial\left(u_{1}, u_{2}, \ldots, u_{n-1}\right)}=t^{2-n} .
$$

So if we take $t$ and $u^{\prime}$ as new variables in place of $u_{1}, u_{2}, \ldots, u_{n}$, the integral is transformed into

$$
\int_{E^{n-1}} \prod_{i \neq j}\left(u_{i}^{\prime}\right)^{s+\alpha_{i}-1} d U^{\prime} \int_{0}^{h} t^{(n-1) s+|\alpha|_{j}-1}(1-t)^{s+\alpha_{j}-1} d t .
$$

The first integral in the above is the standard $\beta$-function of several variables; it is equal to

$$
\prod_{i \neq j} \Gamma\left(s+\alpha_{i}\right) \cdot \frac{1}{\Gamma\left((n-1) s+|\alpha|_{j}\right)} .
$$

Our assertion thus follows. 
We are now ready to evaluate the integral $F(s)$ at $s=1-m$ when $p_{j}=0$ for some $j$. With a permutation in the indices, we can suppose that

$$
p_{1}=\ldots=p_{q}=0, \quad p_{q+1} \geq 1, \ldots, p_{r} \geq 1, \quad 1 \leq q \leq r
$$

For Re $s>0$, set

$$
G(s)=\frac{1}{\Gamma(s)^{n-1}} \int_{E^{n}}\left(u_{1} \ldots u_{n}\right)^{s-1} \prod_{j=q+1}^{r} L_{j}(u)^{p_{j}-1} \prod_{i=1}^{q} L_{i}(u)^{-1} d U
$$

with $p_{q+1}+\ldots+p_{r}=n(m-1)+r$. Note that $\prod_{j=q+1}^{r} L_{j}(u)^{p_{j}-1}$ is a homogeneous polynomial in $u_{1}, \ldots, u_{n}$ of degree $n(m-1)+q$. Put

$$
\prod_{j=q+1}^{r} L_{j}(u)^{p_{j}-1}=\sum_{|\alpha|=n(m-1)+q} b_{\alpha} u_{1}^{\alpha_{1}} \ldots u_{n}^{\alpha_{n}} .
$$

Then

$$
G(s)=\sum_{|\alpha|=n(m-1)+q} b_{\alpha} G_{\alpha}(s)
$$

with

$$
G_{\alpha}(s)=\frac{1}{\Gamma(s)^{n-1}} \int_{E^{n}} \prod_{i=1}^{n} u_{i}^{s+\alpha_{i}-1} \prod_{i=1}^{q} L_{i}(u)^{-1} d U .
$$

For $\operatorname{Re} s>0, G_{\alpha}(s)$ is an analytic function of $s$, and it has analytic continuation in the whole complex plane as we shall see. We are interested in the special value $G_{\alpha}(1-m)$.

Lemma 3. Suppose $\alpha=\left(\alpha_{1}, \ldots, \alpha_{n}\right)$ is an $n$-tuple of nonnegative integers with $|\alpha|=n(m-1)+q, q \geq 1$. If $\alpha_{j}, \alpha_{k} \geq m$ for $j \neq k$, then $G_{\alpha}(1-m)=0$.

Proof. Since the singularities of the integral $G_{\alpha}(s)$ arise from integration around vertices of $E^{n}$, to show $G_{\alpha}(1-m)=0$, it suffices to prove that the partial integration

$$
G_{\alpha}^{p}(s)=\frac{1}{\Gamma(s)^{n-1}} \int_{E_{p}^{n}(h)} \prod_{i=1}^{n} u_{i}^{s+\alpha_{i}-1} \prod_{i=1}^{q} L_{i}(u)^{-1} d U
$$

vanishes at $s=1-m$. But this follows from the fact that the difference

$$
G_{\alpha}(s)-\sum_{p=1}^{n} G_{\alpha}^{p}(s)
$$

can be eliminated via $\Gamma(s)^{1-n}$ when $s=1-m$. In particular, one has

$$
G_{\alpha}(1-m)=\sum_{p=1}^{n} G_{\alpha}^{p}(1-m) .
$$


Around the vertex $u_{p}=1$, suppose that

$$
\prod_{i=1}^{q} L_{i}(u)^{-1}=\sum_{|\beta|=0}^{\infty} C_{\beta} u_{1}^{\beta_{1}} \ldots u_{p-1}^{\beta_{p-1}} u_{p+1}^{\beta_{p+1}} \ldots u_{n}^{\beta_{n}}
$$

By Lemma 2 and a term by term integration, we get

$$
G_{\alpha}^{p}(s)=\sum_{|\beta|=0}^{\infty} C_{\beta} \prod_{i \neq p} \Gamma\left(s+\alpha_{i}+\beta_{i}\right) \cdot \Gamma(s)^{1-n} \cdot I_{\alpha, \beta, p}(s)
$$

where

$$
I_{\alpha, \beta, p}(s)=\frac{1}{\left.\Gamma(n-1) s+|\alpha|_{p}+|\beta|\right)} \int_{0}^{h} t^{(n-1) s+|\alpha|_{p}+|\beta|-1}(1-t)^{s+\alpha_{p}-1} d t .
$$

$I_{\alpha, \beta, p}(s)$ is an analytic function of $s$ for $\operatorname{Re} s>0$ and it has analytic continuation in the whole complex plane. Note that $\Gamma(s)^{1-n}$ has a zero of order $n-1$ at $s=1-m$ while the order of the pole at $s=1-m$ of $\prod_{i \neq p} \Gamma\left(s+\alpha_{i}+\beta_{i}\right)$ is no greater than $n-2$ since $\alpha_{i} \geq m$ for at least one $i$. So $G_{\alpha}^{p}(1-m)=0$ for all $p$. This proves our assertion.

By the above lemma, we know that $G_{\alpha}(1-m)=0$ unless $\alpha_{j} \geq m$ for exactly one $j$. Now we proceed to consider these exceptional cases.

Lemma 4. Suppose that $\alpha=\left(\alpha_{1}, \ldots, \alpha_{n}\right)$ is an n-tuple of non-negative integers such that $|\alpha|=n(m-1)+q, q \geq 1, \alpha_{1}, \ldots, \alpha_{n-1} \leq m-1$ and $\alpha_{n} \geq m$. Then

$$
\begin{aligned}
& G_{\alpha}(1-m)=(-1)^{(n-1)(m-1)}[(m-1) !]^{n-1} \\
& \quad \times \text { the coefficient of } \prod_{i=1}^{n-1} u_{i}^{m-1-\alpha_{i}} \text { in the Taylor expansion } \\
& \text { of the function }\left(1-u_{1}-\ldots-u_{n-1}\right)^{\alpha_{n}-m} \prod_{i=1}^{q} L_{i}(u)^{-1} .
\end{aligned}
$$

Proof. Suppose that around $u_{j}=1$, the Taylor expansion of $\prod_{i=1}^{q} L_{i}(u)^{-1}$ is given by

$$
\sum_{|\beta|=0}^{\infty} C_{\beta} u_{1}^{\beta_{1}} \ldots u_{j-1}^{\beta_{j-1}} u_{j+1}^{\beta_{j+1}} \ldots u_{n}^{\beta_{n}} .
$$

As in the previous lemma, we have for $\operatorname{Re} s>0$,

$$
\begin{aligned}
G_{\alpha}^{j} & =\frac{1}{\Gamma(s)^{n-1}} \int_{E_{j}^{n}(h)} \prod_{i=1}^{n} u_{i}^{s+\alpha_{i}-1} \prod_{i=1}^{q} L_{i}(u)^{-1} d U \\
& =\sum_{|\beta|=0}^{\infty} C_{\beta} \prod_{i \neq j} \Gamma\left(s+\alpha_{i}+\beta_{i}\right) \cdot \Gamma(s)^{1-n} \cdot I_{\alpha, \beta, j}(s),
\end{aligned}
$$


where

$$
I_{\alpha, \beta, j}(s)=\frac{1}{\Gamma\left((n-1) s+|\alpha|_{j}+|\beta|\right)} \int_{0}^{h} t^{(n-1) s+|\alpha|_{j}+|\beta|-1}(1-t)^{s+\alpha_{j}-1} d t .
$$

If $j \neq n$, then the product of gamma functions $\gamma(s)=\prod_{i \neq j} \Gamma\left(s+\alpha_{i}+\beta_{i}\right) \cdot \Gamma(s)^{1-n}$ will vanish at $s=1-m$. So only $G_{\alpha}^{n}(s)$ has a nontrivial contribution to $G_{\alpha}(s)$ at $s=1-m$. In the case $j=n$, the factor $(1-t)^{s+\alpha_{n}-1}$ in $I_{\alpha, \beta, n}(s)$ has no further pole at $t=1$, since $\alpha_{n} \geq m$. So we can replace $I_{\alpha, \beta, n}(s)$ by

$$
\begin{aligned}
& \frac{1}{\Gamma\left((n-1) s+|\alpha|_{n}+|\beta|\right)} \int_{0}^{1} t^{(n-1) s+|\alpha|_{n}+|\beta|-1}(1-t)^{s+\alpha_{n}-1} d t \\
& \quad=\frac{\Gamma\left(s+\alpha_{n}\right)}{\Gamma(n s+|\alpha|+|\beta|)} .
\end{aligned}
$$

In fact, the difference between the two integrals can be eliminated by the zero at $s=1-m$ of $\Gamma(s)^{1-n}$. Consequently, we have

$$
G_{\alpha}^{n}(1-m)=\sum_{|\beta|=0}^{\infty} C_{\beta} A_{\alpha, \beta}(1-m)
$$

where

$$
A_{\alpha, \beta}(s)=\frac{\Gamma\left(s+\alpha_{n}\right) \prod_{i=1}^{n-1} \Gamma\left(s+\alpha_{i}+\beta_{i}\right)}{\Gamma(s)^{n-1} \Gamma(n s+|\alpha|+|\beta|)} .
$$

Note that $A_{\alpha, \beta}(s)$ is an analytic function of $s$ for $\operatorname{Re} s>0$ and it has analytic continuation in the whole complex s-plane. Also $A_{\alpha, \beta}(1-m)=0$ unless $\alpha_{i}+\beta_{i} \leq$ $m-1$ for $1 \leq i \leq n-1$. For such exceptional cases, one has

$$
A_{\alpha, \beta}(1-m)=(-1)^{|\alpha|_{n}+|\beta|} \frac{\left(\alpha_{n}-m\right) !}{(\beta+q-1) !} \prod_{i=1}^{n-1} \frac{(m-1) !}{\left(m-1-\alpha_{i}-\beta_{i}\right) !} .
$$

It follows that

$$
\begin{aligned}
& G_{\alpha}(1-m)=G_{\alpha}^{n}(1-m) \\
& \quad=\sum_{0 \leq \beta_{i} \leq m-1-\alpha_{i}}(-1)^{|\alpha|_{n}+|\beta|} C_{\beta} \frac{\left(\alpha_{n}-m\right) !}{(\beta+q-1) !} \prod_{i=1}^{n-1} \frac{(m-1) !}{\left(m-1-\alpha_{i}-\beta_{i}\right) !} \\
& \quad=(-1)^{(n-1)(m-1)}[(m-1) !]^{n-1} \times \text { the coefficient of } \prod_{i=1}^{n-1} u_{i}^{m-1-\alpha_{i}} \text { in the } \\
& \text { Taylor expansion of the function }\left(1-u_{1}-\ldots-u_{n-1}\right)^{\alpha_{n}-m} \prod_{j=1}^{q} L_{j}(u)^{-1}
\end{aligned}
$$

With Lemma 3 and 4, we are able to find the special value $G(1-m)$ in terms of coefficients of functions

$$
u_{j}^{-m} \prod_{i=q+1}^{r} L_{i}(u)^{p_{i}-1} / \prod_{i=1}^{q} L_{i}(u) \quad(j=1, \ldots, n) .
$$


This is equivalent to compute Shintani's coefficients $C(s, q, A) \quad(k=1, \ldots, n)$, which are defined in terms of coefficients of the function

$$
\prod_{i=q+1}^{r} L_{i}(u)^{p_{i}-1} /\left.\prod_{i=1}^{q} L_{i}(u)\right|_{u_{j}=1}
$$

Precisely, we have the following.

Lemma 5. Let

$$
f_{p}(u)=\prod_{i=q+1}^{r} L_{i}(u)^{p_{i}-1} / \prod_{i=1}^{q} L_{i}(u)
$$

with $|p|=n(m-1)+r$. Then the coefficient of $\left(u_{1} \ldots u_{n-1}\right)^{m-1}$ in the Taylor expansion of $f_{p}\left(u_{1}, \ldots, u_{n-1}, 1\right)$ at $u_{1}=\ldots=u_{n-1}=0$ is equal to the coefficient of $\left(u_{1}, \ldots, u_{n-1}\right)^{m-1}$ in the Taylor expansion of

$$
g\left(u_{1}, \ldots, u_{n-1}\right)=\left(1-u_{1}-\cdots-u_{n-1}\right)^{-m} f\left(u_{1}, \ldots, u_{n-1}, 1-u_{1}-\ldots-u_{n-1}\right)
$$

at $u_{1}=\ldots=u_{n-1}=0$.

Proof. For Re $s>0$, consider the integral

$$
G(s)=\frac{1}{\Gamma(s)^{n-1}} \int_{E^{n}}\left(u_{1} \ldots u_{n}\right)^{s-1} f_{p}(u) d U .
$$

For $1 \leq j \leq n$, we let $F_{j}$ be the subset of $E^{n}$ defined by

$$
F_{j}=\left\{u=\left(u_{1}, \ldots, u_{n}\right) \in E^{n} \mid u_{i} \leq u_{j} \text { for all } i \neq j\right\} .
$$

Then $F_{1}, \ldots, F_{n}$ are pairwise non-overlapping and $E^{n}=\bigcup_{j=1}^{n} F_{j}$. Let

$$
G_{j}(s)=\frac{1}{\Gamma(s)^{n-1}} \int_{F_{j}}\left(u_{1} \ldots u_{n}\right)^{s-1} f_{p}(u) d U
$$

Now we focus our attention on the case $j=n$. $F_{n}$ contains the vertex $V_{n}=$ $(0, \ldots, 0,1)$ of $E_{n}$, so by Lemma 4 , we have

$$
\begin{aligned}
G_{n}(1-m)= & (-1)^{(n-1)(m-1)}[(m-1) !]^{n-1} \\
& \times \text { the coefficient of }\left(u_{1} \ldots u_{n-1}\right)^{m-1} \text { in the Taylor expansion of } \\
& g\left(u_{1}, \ldots, u_{n-1}\right) \text { at } u_{1}=\ldots=u_{n-1}=0 .
\end{aligned}
$$

We want to prove $G_{n}(1-m)$ can also be given by the coefficient of $f_{p}\left(u_{1}, \ldots, u_{n-1}, 1\right)$. With the change of variables: $\left(u_{1}, \ldots, u_{n}\right)=u\left(v_{1}, \ldots, v_{n-1}, 1\right)$, we then have

$$
G_{n}(s)=\frac{1}{\Gamma(s)^{n-1}} \int_{1 / n}^{1} u^{n s+n(m-1)-2} d u \int_{C(u)}\left(v_{1} \ldots v_{n-1}\right)^{s-1} f_{p}\left(v_{1}, \ldots, v_{n-1}\right) d V
$$

where $C(u)$ is the simplex in $\mathbb{R}^{n-1}$ defined by

$$
C(u)=\left\{\left(v_{1}, \ldots, v_{n-1}\right) \mid 0 \leq v_{i} \leq 1, v_{1}+\ldots+v_{n-1}=(1-u) / u\right\} .
$$


Observe that the singularities of $G_{n}(s)$ arise from the integration around $u=1$. In order to get $G_{n}(1-m)$, we consider

$$
\begin{aligned}
G_{n, q}(s)= & \frac{1}{\Gamma(s)^{n-1}} \int_{1-\delta}^{1} u^{n s+n(m-1)-2} d u \\
& \times \int_{C(u)}\left(v_{1} \ldots v_{n-1}\right)^{s-1} f_{p}\left(v_{1}, \ldots, v_{n-1}, 1\right) d V
\end{aligned}
$$

instead for sufficiently small $\delta>0$. For $v_{1}+\ldots+v_{n-1}<\frac{\delta}{1-\delta}$, suppose that

$$
f_{p}\left(v_{1}, \ldots, v_{n-1}, 1\right)=\sum_{|\alpha|=0}^{\infty} b_{\alpha} v_{1}^{\alpha_{1}} \ldots v_{n-1}^{\alpha_{n-1}}
$$

With the change of variable $v_{i}=\frac{1-u}{u} v_{i}^{\prime}(i=1, \ldots, n-1)$ and a term by term integration, it yields for $\operatorname{Re} s>0$ that

$$
\begin{aligned}
G_{n, \delta}(s)= & \sum_{|\alpha|=0}^{\infty} b_{\alpha} \frac{\Gamma\left(s+\alpha_{1}\right) \cdots \Gamma\left(s+\alpha_{n-1}\right)}{\Gamma((n-1) s+|\alpha|) \Gamma(s)^{n-1}} \\
& \times \int_{1-\delta}^{1}(1-u)^{(n-1) s+|\alpha|-1} u^{s+n(m-1)-|\alpha|-1} d u .
\end{aligned}
$$

If $|\alpha|>(n-1)(m-1)$, the integrals appear in $G_{n, \delta}(s)$ are convergent for $s=1-m$ while the product of gamma functions vanishes at $s=1-m$. On the other hand, if $|\alpha|<(n-1)(m-1)$, then $u^{s+n(m-1)-|\alpha|-1}$ is a regular function of $u$ at $s=1-m$ and $u=0$, so one can replace the integral in $G_{n, \delta}(s)$ by

$$
\begin{aligned}
\int_{0}^{1}(1-u)^{(n-1) s+|\alpha|-1} u^{s+n(m-1)-|\alpha|-1} d u \\
=\frac{\Gamma((n-1) s+|\alpha|) \Gamma(s+n(m-1)-|\alpha|)}{\Gamma(n s+n(m-1))}
\end{aligned}
$$

Hence the contribution to $G_{n, \delta}(1-m)$ from those terms with $|\alpha|<(n-1)(m-1)$ is given by

$$
\sum_{|\alpha|<(n-1)(m-1)} b_{\alpha} a_{\alpha}(1-m)
$$

where

$$
a_{\alpha}(s)=\frac{\Gamma\left(s+\alpha_{1}\right) \ldots \Gamma\left(s+\alpha_{n-1}\right) \Gamma(s+n(m-1)-|\alpha|)}{\Gamma(s)^{n-1} \Gamma(n s+n(m-1))} .
$$

However $a_{\alpha}(1-m)=0$. It remains to consider the cases $|\alpha|=(n-1)(m-1)$. In these case $\frac{\Gamma\left(s+\alpha_{1}\right) \ldots \Gamma\left(s+\alpha_{n-1}\right)}{\Gamma(s)^{n-1}}$ vanishes at $s=1-m$ unless $\alpha_{1}=\cdots=$ $\alpha_{n-1}=m-1$. Also the special value at $s=1-m$ of the integral

$$
\frac{1}{\Gamma((n-1) s+(n-1)(m-1))} \int_{1-\delta}^{1}(1-u)^{(n-1)(s+m-1)-1} u^{s+m-2} d u
$$


is 1 by the residue theorem. Consequently we have

$$
G_{n, \delta}(1-m)=(-1)^{(n-1)(m-1)}[(m-1) !]^{n-1} b_{m-1, \ldots, m-1} .
$$

This proves our assertion.

Integrals of types $F(s)$ and $G(s)$ are considered in [4] with the notation $H_{\beta}(s)$ :

$$
H_{\beta}(s)=\frac{1}{\Gamma(s)^{n-1}} \int_{E^{n}}\left(u_{1} \ldots u_{n}\right)^{s-1} \prod_{j=1}^{r}\left[L_{j}(u)\right]^{\beta_{j}-1} d U
$$

where $\beta=\left(\beta_{1}, \ldots, \beta_{r}\right)$ is an $r$-tuple of non-negative integers with $|\beta|=n(m-1)+r$. Also the special value $H_{\beta}(1-m)$ is given explicitly there. Now we are ready to prove the theorem.

Proof of the theorem. For Re $s>0$, one has

$$
\begin{aligned}
\Gamma(s)^{n} \prod_{k=1}^{n}\left[a _ { 1 k } \left(n_{1}\right.\right. & \left.\left.+x_{1}\right)+\ldots+a_{r k}\left(n_{r}+x_{r}\right)\right]^{-s}=\int_{0}^{\infty} \ldots \int_{0}^{\infty}\left(y_{1} \ldots y_{n}\right)^{s-1} \\
& \times \exp \left[-\sum_{k=1}^{n}\left[a_{1 k}\left(n_{1}+x_{1}\right)+\ldots+a_{r k}\left(n_{r}+x_{r}\right)\right] y_{k}\right] d y_{1} \ldots d y_{n}
\end{aligned}
$$

Therefore for Re $s>r / n$, one has

$$
\zeta(s, A, x) \Gamma(s)^{n}=\int_{0}^{\infty} \ldots \int_{0}^{\infty}\left(y_{1} \ldots y_{n}\right)^{s-1} \prod_{j=1}^{r} \frac{\exp \left[\left(1-x_{j}\right) L_{j}(y)\right]}{\exp \left[L_{j}(y)\right]-1} d y_{1} \ldots d y_{n} .
$$

With the change of variables

$$
\left(y_{1}, \ldots, y_{n}\right)=t\left(u_{1}, \ldots, u_{n}\right), \quad t>0,\left(u_{1}, \ldots, u_{n}\right) \in E^{n},
$$

the integral becomes

$$
\zeta(s, A, x) \Gamma(s)^{n}=\int_{0}^{\infty} t^{n s-1} d t \int_{E^{n}}\left(u_{1}, \ldots, u_{n}\right)^{s-1} \prod_{j=1}^{r} \frac{\exp \left[t\left(1-x_{j}\right) L_{j}(u)\right]}{\exp \left[t L_{j}(u)\right]-1} d U .
$$

Rewrite the above formula as

$$
\zeta(s, A, x) \Gamma(s)=\int_{0}^{\infty} t^{n s-r-1} I_{n}(s, t, x) d t
$$

where

$$
I_{n}(s, t, x)=\frac{1}{\Gamma(s)^{n-1}} \int_{E^{n}}\left(u_{1} \ldots u_{n}\right)^{s-1} \prod_{j=1}^{r} \frac{t \exp \left[t\left(1-x_{j}\right) L_{j}(u)\right]}{\exp \left[t L_{j}(u)\right]-1} d U .
$$

$I_{n}(s, t, x)$ is an analytic function of $s$ for $\operatorname{Re} s>0$ and has an analytic continuation since it is an integration of a continuous function over $E_{n}$ with respect to the measure

$$
\frac{1}{\Gamma(s)^{n-1}}\left(u_{1} \ldots u_{n}\right)^{s-1} d U
$$


On the other hand, $I_{n}(s, t, x)$ is a rapidly decreasing function in $t$, and for sufficiently small $t$, it has the power series expansion

$$
\sum_{|\beta|=0}^{\infty} t^{|\beta|} \frac{B_{\beta_{1}}\left(1-x_{1}\right) \ldots B_{\beta_{r}}\left(1-x_{r}\right)}{\beta_{1} ! \ldots \beta_{r} !} H_{\beta}(s) .
$$

Now the special value of $\zeta(s, A, x)$ at $s=1-m$ can be determined by comparing the residues at $s=1-m$ in both sides of equation

$$
\zeta(s, A, x) \Gamma(s)=\int_{0}^{\infty} t^{n s-r-1} I_{n}(s, t, x) d t .
$$

The result is

$\zeta(1-m, A, x)=\frac{(-1)^{m-1}(m-1) !}{n} \sum_{|\beta|=n(m-1)+r} \frac{(-1)^{\beta} B_{\beta_{1}}\left(x_{1}\right) \cdots B_{\beta_{r}}\left(x_{r}\right)}{\beta_{1} ! \cdots \beta_{r} !} H_{\beta}(1-m)$.

For $\beta_{1} \geq 1, \cdots, \beta_{r} \geq 1, \prod_{j=1}^{r}\left[L_{j}(u)\right]^{\beta_{j}-1}$ is a homogeneous polynomial in $u_{1}, \cdots, u_{n}$ of degree $n(m-1)$. By Proposition 4 of [4], we have

$$
\frac{(-1)^{m-1}(m-1) !}{n} \sum_{\substack{|\beta|=n(m-1)+r \\ \beta_{1} \geq 1, \cdots, \beta_{r} \geq 1}} \frac{(-1)^{\beta} B_{\beta_{1}}\left(x_{1}\right) \cdots B_{\beta_{r}}\left(x_{r}\right)}{\beta_{1} ! \cdots \beta_{r} !} H_{\beta}(1-m)=J_{I}\left[P^{m-1}(u)\right],
$$

where $I=\{1,2, \cdots, r\}$ and $P(u)=\prod_{j=1}^{n}\left(a_{1 j} u_{1}+\cdots+a_{r j} u_{r}\right)$.

If $\beta_{1}=\cdots=\beta_{q}=0, \beta_{q+1} \geq 1, \cdots, \beta_{r} \geq 1$, we let $S=\{q+1, \cdots, r\}$. By Propositions 5 and 6 of [4], we get

$$
\begin{gathered}
\frac{(-1)^{m-1}(m-1) !}{n} \quad \sum_{\substack{|\beta|=n(m-1)+r \\
\beta_{1}=\cdots=\beta_{q}=0, \beta_{q+1} \geq 1, \cdots, \beta_{r} \geq 1}} \frac{(-1)^{\beta} B_{\beta_{q+1}}\left(x_{q+1}\right) \cdots B_{\beta_{r}}\left(x_{r}\right)}{\beta_{q+1} ! \cdots \beta_{r} !} H_{\beta}(1-m) \\
=\frac{1}{n} J_{S}\left[\sum_{j=1}^{n} \int_{\Delta_{j, S}(u)} P^{m-1}(u) d u_{1} \cdots d u_{q}\right],
\end{gathered}
$$

where $\Delta_{j, S}(u)$ is the simplex in $\mathbb{R}^{q}$ defined by

$$
\Delta_{j, S}(u):\left\{\begin{array}{l}
\left(u_{1}, \cdots, u_{q}\right) \in \mathbb{R}^{q}, \\
u_{1} \leq 0, \cdots, u_{q} \leq 0, \quad L_{j}(u)=a_{1 j} u_{1}+\cdots+a_{r j} u_{r} \geq 0 .
\end{array}\right.
$$

We now compare our formula with the formula obtained by Shintani in [6]. First we consider the coefficient $C(A, p)$ that appears in the first part of Shintani's formula for $\zeta(1-m, A, x)$. According to his result, it is the coefficient of $\left(t_{1} \ldots t_{n}\right)^{m-1}$ in the polynomial function $\prod_{j=1}^{r} L_{j}(t)^{p_{j}-1}$. As we obtained in Lemma 1 , it is the special value of the integral

$$
F(s)=\frac{1}{\Gamma(s)^{n-1}} \int_{E^{n}}\left(u_{1} \ldots u_{n}\right)^{s-1} \prod_{j=1}^{r} L_{j}(u)^{p_{j}-1} d U
$$


up to a constant factor. Indeed, we have

$$
n C(A, p)=(-1)^{(n-1)(m-1)}[(m-1) !]^{n-1} F(1-m) .
$$

However we have the further result that $C(A, p)$ is nothing but the coefficient of

$$
\frac{(-1)^{(n(m-1))}}{\left(p_{1}-1\right) ! \ldots\left(p_{r}-1\right) !} u_{1}^{p_{1}-1} \ldots u_{r}^{p_{r}-1}
$$

in the polynomial function

$$
\prod_{k=1}^{n}\left[L_{k}^{*}(u)\right]^{m-1}=\prod_{k=1}^{n}\left[a_{1 k} u_{1}+\ldots+a_{r k} u_{r}\right]^{m-1} .
$$

In fact, if

$$
\prod_{k=1}^{n}\left[L_{k}^{*}(u)\right]^{m-1}=\sum_{|\alpha|=n(m-1)} C_{\alpha} u_{1}^{\alpha_{1}} \ldots u_{r}^{\alpha_{r}}
$$

then

$$
\begin{aligned}
& \sum_{|p|=n(m-1)+r} \frac{B_{p_{1}}\left(x_{1}\right) \ldots B_{p_{r}}\left(x_{r}\right)}{p_{1} ! \ldots p_{r} !} C(A, p) \\
= & \sum_{|\alpha|=n(m-1)} C_{\alpha}(-1)^{n(m-1)} \frac{B_{\alpha_{1}+1}\left(x_{1}\right) \ldots B_{\alpha_{r}+1}\left(x_{r}\right)}{\left(\alpha_{1}+1\right) \ldots\left(\alpha_{r}+1\right)} .
\end{aligned}
$$

Secondly, consider the coefficient $C(S, q, A)^{(k)}(k=1, \ldots, n)$. It is the coefficient of $\left(t_{1} \ldots t_{k-1} t_{k+1} \ldots t_{n}\right)^{m-1}$ in the Taylor expansion of the function

$$
\prod_{j \in S} L_{j}(t)^{q(j)-1} /\left.\prod_{j \notin S} L_{j}(t)\right|_{t_{k}=1}
$$

Again we rewrite such a coefficient as the special value of the integral

$$
G(s)=\frac{1}{\Gamma(s)} \int_{E^{n}}\left(u_{1} \ldots u_{n}\right)^{s-1} \prod_{j \in S} L_{j}(u)^{q(j)-1} / \prod_{j \notin S} L_{j}(u) d U .
$$

The special value of $G(s)$ at $1-m$ can be obtained by computing the coefficient of certain functions expanded at vertices of $E^{n}$. The difficulty in such an algorithm is the discovery that these coefficients are also coefficients of polynomials which can be obtained from $\prod_{k=1}^{n}\left[L_{k}^{*}(u)\right]^{m-1}$ in an elementary way. For example, for the case $S=\{q+1, \ldots, r\}$, the polynomial function is given by

$$
\frac{1}{n} \sum_{j=1}^{n} \int_{\Delta_{j}(u)} \prod_{k=1}^{n}\left[L_{k}^{*}(u)\right]^{m-1} d u_{1} \ldots d u_{q},
$$

where $\Delta_{j}(u)$ is the simplex in $\mathbb{R}^{q}$ defined by

$$
\Delta_{j}(u): u_{1} \leq 0, \ldots, u_{q} \leq 0, \quad L_{j}^{*}(u) \geq 0 .
$$




\section{Identities Among Bernoulli polynomials}

Here we discuss two kinds of identities among Bernoulli polynomials.

Proposition 2. For a positive integer $n \geq 2$, one has

$$
\begin{aligned}
& \sum_{k=1}^{2 n-1} \frac{(2 n) !}{k !(2 n-k) !} B_{k}(\alpha) B_{2 n-k}(\beta) \\
& \quad=-\left[(2 n-1) B_{2 n}(\alpha+\beta)+2 n(1-\alpha-\beta) B_{2 n-1}(\alpha+\beta)+B_{2 n}(\alpha)+B_{2 n}(\beta)\right] .
\end{aligned}
$$

Proof. For the time being, we assume that $\alpha$ and $\beta$ are positive numbers. For $\operatorname{Re} s>1$, consider the identity of zeta functions

$$
\begin{aligned}
\sum_{n_{1}=0}^{\infty} \sum_{n_{2}=0}^{\infty}\left[\left(n_{1}+\alpha\right)+\left(n_{2}+\beta\right)\right]^{-s} & =\sum_{k=0}^{\infty} \frac{k+1}{k+\alpha+\beta} \\
& =\zeta(s-1 ; \alpha+\beta)+(1-\alpha-\beta) \zeta(s ; \alpha+\beta) .
\end{aligned}
$$

From the special value at $s=2-2 n$, we get

$$
\begin{aligned}
& \sum_{k=1}^{2 n-1} \frac{(2 n-2) !}{k !(2 n-k) !} B_{k}(\alpha) B_{2 n-k}(\beta)+\frac{1}{(2 n-1) 2 n} B_{2 n}(\alpha)+\frac{1}{(2 n-1) 2 n} B_{2 n}(\beta) \\
& =-\frac{1}{2 n} B_{2 n}(\alpha+\beta)-\frac{1-\alpha-\beta}{2 n-1} B_{2 n-1}(\alpha+\beta) .
\end{aligned}
$$

Multiplying both sides by $2 n(2 n-1)$, we get our assertion for $\alpha>0$ and $\beta>0$. Both sides of the identity are polynomial functions in $\alpha$ and $\beta$; they are equal for $\alpha>0$ and $\beta>0$. It follows that they are equal for all $\alpha$ and $\beta$.

Proposition 3. For a positive integer $n>1$, one has

$$
\begin{aligned}
& \sum_{k=1}^{n-1} \frac{(2 n) !}{(2 k) !(2 n-2 k) !} 4^{2 k} 6^{2 n-2 k} B_{2 k} B_{2 n-2 k} \\
& \quad=-\left[(2 n-1) 2^{2 n}+6^{2 n}+4^{2 n}\right] B_{2 n}+(16 n) 6^{2 n-2} B_{2 n}\left(\frac{1}{3}\right) .
\end{aligned}
$$

Proof. Consider the zeta function

$$
Z(s)=\sum_{n_{1}=1}^{\infty} \sum_{n_{2}=1}^{\infty}\left(4 n_{1}+6 n_{2}\right)^{-s}+\left(4^{-s}+6^{-s}\right) \zeta(s)
$$

which is equal to the Dirichlet series

$$
\sum_{k=1}^{\infty} a(k) k^{-s}
$$

where $a(k)$ is the number of non-negative integral solutions of the equation $4 x+6 y=$ $k$. Obviously, $a(k)=0$ if $k$ is odd. For even $k$, we have

$$
a(k)=\frac{k+5}{12}+\frac{1}{4} \delta_{1}(k)+\frac{1}{3} \delta_{2}(k),
$$


with

$$
\delta_{1}(k)=\left\{\begin{array}{ll}
1 & \text { if } k \equiv 0(\bmod 4), \\
-1 & \text { if } k \equiv 2(\bmod 4),
\end{array} \quad \delta_{2}(k)= \begin{cases}1 & \text { if } k \equiv 0(\bmod 6) \\
-1 & \text { if } k \equiv 2(\bmod 6) \\
0 & \text { if } k \equiv 4(\bmod 6)\end{cases}\right.
$$

Consequently, from two expressions of $Z(2-2 n)$, we get

$$
\begin{aligned}
& \sum_{k=1}^{n-1} \frac{(2 n) !}{(2 k) !(2 n-2 k) !} 4^{2 k} 6^{2 n-2 k} B_{2 k} B_{2 n-2 k} \\
& =-\left[(2 n-1) 2^{2 n}+6^{2 n}+4^{2 n}\right] B_{2 n}+(3 n) 4^{2 n-1} B_{2 n-1}\left(\frac{1}{2}\right)+(16 n) 6^{2 n-2} B_{2 n}\left(\frac{1}{3}\right)
\end{aligned}
$$

Note that $B_{2 n-1}\left(\frac{1}{2}\right)=0$. So we have the desired identity.

Remark. $a(k)$ is just the dimension of the space of modular forms of weight $k$ of one variable in the upper half plane.

\section{Generalized Bernoulli numbers}

Let $\chi$ be a Dirichlet character with conductor $N$. The $L$-series defined by

$$
L(s ; \chi)=\sum_{n=1}^{\infty} \chi(n) n^{-s}, \quad \operatorname{Re} s>1,
$$

is a holomorphic function of $s$ for $\operatorname{Re} s>1$. Also it has an analytic continuation which is holomorphic in the whole complex plane except for a possible simple pole at $s=1$ when $\chi$ is the trivial character $\chi_{0}$ defined by

$$
\chi_{0}(n)=1 \quad \text { if }(n, N)=1
$$

Especially, the special value of $L(s ; \chi)$ at a non-positive integer $-m$ is given by

$$
L(-m ; \chi)=-\frac{B_{m+1, \chi}}{m+1}
$$

where the generalized Bernoulli number $B_{m, \chi}$ is defined by

$$
\left(\sum_{j=1}^{N} \chi(j) e^{j t}\right) t\left(e^{N t}-1\right)^{-1}=\sum_{m=0}^{\infty} \frac{t^{m}}{m !} B_{m, \chi}, \quad|t|<\frac{2 \pi}{N} .
$$

See also [9] for the definition. From the definition, we see immediately that

$$
B_{m+1, \chi}=N^{m} \sum_{j=1}^{N} \chi(j) B_{m+1}\left(\frac{j}{N}\right) .
$$

In the following, we compute the special values of an $L$-series by the result of the author in [3]. 
Proposition 4. Suppose that $\chi$ is not a trivial character and

$$
P(x)=\prod_{j=1}^{k}\left(a_{j} x+\delta_{j}\right), \quad a_{j}>0, \operatorname{Re} \delta_{j} / a_{j}>-1,
$$

is a polynomial with real coefficients. Define

$$
L(s ; P, \chi)=\sum_{n=1}^{\infty} \chi(n) P(n)^{-s}, \quad \operatorname{Re} s>1 / k .
$$

Then for any integer $m \geq 0$,

$$
L(-m ; P, \chi)=\sum_{r=0}^{k m} a_{r}\left(-\frac{B_{r+1, \chi}}{r+1}\right)
$$

where $a_{r}$ is the coefficient of $x^{r}$ in $P^{m}(x)$.

Proof. Replace $P(x)$ by $P^{m}(x)$ if necessary; we therefore need only consider the cases $m=0$ and $m=1$. Rewrite the zeta function as

$$
L(s ; P, \chi)=\sum_{j=1}^{N} \chi(j) \sum_{n=0}^{\infty} P(j+n N)^{-s}
$$

Set

$$
P(x)=\sum_{i=0}^{k} b_{i} x^{i} \quad \text { and } \quad Z_{P, j}(s)=\sum_{n=0}^{\infty} P(j+n N)^{-s} .
$$

Then by the main theorem of [3], we have

$$
Z_{P, j}(-1)=P(j)+J[P(j+N x)]+\frac{1}{k} \sum_{i=1}^{k} \int_{0}^{\mu_{i j}} P(j+N x) d x,
$$

where $\mu_{i j}=-\left(j a_{i}+\delta_{j}\right) / a_{i} N(i=1, \cdots, k)$ are zeros of $P(j+N x)$. By a change of variable in the integral of $Z_{P, j}(-1)$, we get

$$
\begin{aligned}
Z_{P, j}(-1) & =P(j)+J[P(j+N x)]+\frac{1}{k N} \sum_{i=1}^{k} \int_{j}^{-\delta_{i} / a_{i}} P(x) d x \\
& =P(j)+J[P(j+N x)]+\frac{1}{k N} \sum_{i=1}^{k} \int_{0}^{-\delta_{i} / a_{i}} P(x) d x-\frac{1}{N} \int_{0}^{j} P(x) d x .
\end{aligned}
$$

A direct verification shows that

$$
P(j)+J[P(j+N x)]-\frac{1}{N} \int_{0}^{j} P(x) d x=-\sum_{i=0}^{k} b_{i} \frac{N_{i}}{i+1} B_{i+1}\left(\frac{j}{N}\right) .
$$


It follows that

$$
\begin{aligned}
L(-1 ; P, \chi) & =\sum_{j=1}^{N} \chi(j) Z_{P, j}(-1) \\
& =-\sum_{i=0}^{k} \frac{b_{i}}{i+1} \sum_{j=1}^{N} \chi(j) N^{i} B_{i+1}\left(\frac{j}{N}\right)+\frac{1}{k N} \sum_{j=1}^{N} \chi(j) \sum_{i=1}^{k} \int_{0}^{-\delta_{j} / a_{i}} P(x) d x \\
& =-\sum_{i=0}^{k} \frac{b_{i}}{i+1} B_{i+1, \chi} .
\end{aligned}
$$

In the same way, we can compute the special value $L(0 ; P, \chi)$. This proves our assertion.

\section{ACKNOWLEDGEMENT}

The author wishes to thank one of the referees for encouraging him to rewrite this papar in its present form. He went through the paper thoroughly and carefully, corrected the typos and improved the exposition of the paper a great deal. Thanks are also due to the editor, Professor Winnie Li, who gave the author a useful bit of advice during the preparation of the paper.

\section{REFERENCES}

1. B. C. Berndt, Ramanujan's notebooks, Part I and Part II, Springer-Verlag, 1985 and 1989. MR 86c:01062; MR 90b:01039

2. B. C. Carlson, Special functions of applied mathematics (1977), Academic Press. MR 58:28707

3. Minking Eie, On a Dirichlet series associated with a polynomial, Proceedings of A. M. S. 99 (1990), 583-590. MR 91m:11071

4. Minking Eie, The special values at negative integers of Dirichlet series associated with polynomials of several variables, Proceedings of A. M. S. 119 (1993), 51-61. MR 93h:11082

5. G. van der Geer, Hilbert modular surface (1988), Springer-Verlag. MR 89c:11073

6. T. Shintani, On evaluation of zeta functions of totally real algebraic number fields at nonpositive integers, J. Fac. Sci. University of Tokyo 23 (1976), 393-417. MR 55:266

7. H. Rademacher, Topics in analytic number theory (1973), Springer-Verlag. MR 51:358

8. C. L. Siegel, Lectures on advanced analytic number theory, Tata Institute of Fundamental Research, Bombay, 1965. MR 41:6760

9. L. C. Washington, Introduction to cyclotomic fields, Springer-Verlag, 1982. MR 85g:11001

10. D. Zagier, Valeurs des fontions zêta des corps quadratiques réels aux entiers négatifs, Astérisque 41-42 (1977), 135-151. MR 56:316

Institute of Applied Mathematics, National Chung Cheng University, MingHsiung, Chia-Yi 621, Taiwan, Republic of China.

E-mail address: mkeie@math.ccu.edu.tw 